\title{
Understanding Comprehensive Transcriptional Response of Salmonella enterica spp. in Contact with Cabbage and Napa Cabbage ${ }^{\mathbb{S}}$
}

\author{
Hojun Lee ${ }^{1 \dagger}$, Seul I Kim ${ }^{1 \dagger}$, Sojung Park ${ }^{1}$, Eunwoo Nam ${ }^{1}$, and Hyunjin Yoon ${ }^{1,2 *}$ \\ ${ }^{1}$ Department of Molecular Science and Technology, Ajou University, Suwon 16499, Republic of Korea \\ ${ }^{2}$ Department of Applied Chemistry and Biological Engineering, Ajou University, Suwon 16499, Republic of Korea
}

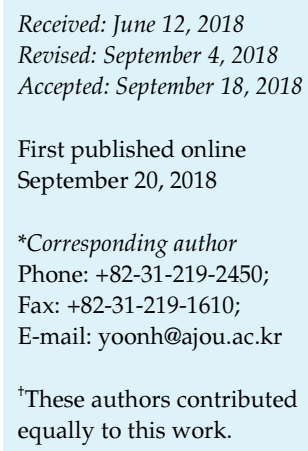

S upplementary data for this paper are available on-line only at http://jmb.or.kr.

pISSN 1017-7825, eISSN 1738-8872

Copyright(C) 2018 by

The Korean Society for Microbiology and Biotechnology

\begin{abstract}
Salmonellosis is commonly associated with meat and poultry products, but an increasing number of Salmonella outbreaks have been attributed to contaminated vegetables and fruits. Enteric pathogens including Salmonella enterica spp. can colonize diverse produce and persist for a long time. Considering that fresh vegetables and fruits are usually consumed raw without heat treatments, Salmonella contamination may subsequently lead to serious human infections. In order to understand the underlying mechanism of Salmonella adaptation to produce, we investigated the transcriptomics of Salmonella in contact with green vegetables, namely cabbage and napa cabbage. Interestingly, Salmonella pathogenicity island (SPI)-1 genes, which are required for Salmonella invasion into host cells, were up-regulated upon contact with vegetables, suggesting that SPI-1 may be implicated in Salmonella colonization of plant tissues as well as animal tissues. Furthermore, Salmonella transcriptomic profiling revealed several genetic loci that showed significant changes in their expression in response to vegetables and were associated with bacterial adaptation to unfavorable niches, including STM14_0818 and STM14_0817 (speF/potE), STM14_0880 (nadA), STM14_1894 to STM14_1892 (fdnGHI), STM14_2006 (ogt), STM14_2269, and STM14_2513 to STM14_2523 (cbi operon). Here, we show that $\operatorname{nadA}$ was required for bacterial growth under nutrient-restricted conditions, while the other genes were required for bacterial invasion into host cells. The transcriptomes of Salmonella in contact with cabbage and napa cabbage provided insights into the comprehensive bacterial transcriptional response to produce and also suggested diverse virulence determinants relevant to Salmonella survival and adaptation.
\end{abstract}

Keywords: Salmonella, vegetable, transcriptome, virulence

\section{Introduction}

Salmonella enterica is a rod-shaped, flagellated, facultative anaerobic, gram-negative bacterial species diverging into more than 2,600 different serovars differentiated by their antigenic presentation [1]. Salmonellae are capable of infecting a wide range of animals including humans and cause diverse diseases from gastroenteritis to typhoid fever. Due to its host preference, plants have been generally presumed not to provide favorable habitats for Salmonella. Indeed, plant environments can be hostile to Salmonella in the context of limited nutrient availability, atmospheric oxygen level on plant surfaces, antimicrobial compounds released by plants, and a large temperature difference between day and night [2]. However, Salmonellae evolutionarily adapted to animal hosts are thought to be excreted back to the soil through the feces of infected hosts, which then eventually contaminate farm produce in the form of composted manure. In this context, Salmonellae exploit farm produce as an environmental reservoir for their persistence and also as a potential vehicle allowing their transmission to their preferable animal hosts [3]. The biggest risk associated with Salmonella-contaminated produce is that fresh and fresh-cut vegetables are usually consumed without being cooked, and thus are likely to cause serious foodborne infection. Furthermore, enteric 
pathogens such as Salmonella are more tolerant toward sanitizing agents such as chlorine [4, 5], increasing the likelihood of enteric pathogens dominating the sanitized produce over indigenous non-pathogenic bacteria [5].

A tremendous research effort has been put into deciphering the underlying mechanism for Salmonella persistence in unfavorable produce environments for the last decade. In the same vein, the aim of this study was to gain insight into how Salmonella orchestrates its transcriptome in response to multiple stimuli from vegetables and further identify genetic loci important for its adaptation to raw produce. S. enterica spp. FORC_015, FORC_019, and FORC_030 used in this study are clinical isolates recovered from human blood and feces, and their genome sequences were recently catalogued in the NCBI database (CP011365.1; CP012396.1; CP015598.1). Serotyping based on KauffmanWhite classification [6] identified these strains as S. enterica serovars Mbandaka, Enteritidis, and Typhimurium, respectively. All three serotypes are closely associated with produce-derived food commodities, according to surveillance in the United States between 1998-2008 [7]. Green leafy vegetables such as lettuce and cabbage are the main cause of produce-associated outbreaks [8]. Microscopic analysis has revealed that Salmonella colonized the surface of vegetable leaves and penetrated into tissues through open stomata [9]. In this study, the three Salmonella strains were exposed to cut cabbage and napa cabbage, and then a transcriptomic analysis was performed to better understand the systematic gene regulation of Salmonella enterica spp. in response to exposure to these vegetables. The transcriptome data were further utilized to identify bacterial determinants required for successful adaptation to cabbage and napa cabbage as an alternative habitat.

\section{Materials and Methods}

\section{Bacterial Strains and Growth Condition}

Salmonella enterica spp. wild-type strains including three FORC strains and S. Typhimurium 14028S, and their isogenic mutant derivatives used in this study are listed in Table S1. Unless stated, all bacterial strains were grown in Luria-Bertani (LB) medium at $37^{\circ} \mathrm{C}$, and the following antibiotics (Sigma-Aldrich, USA) were added when required: kanamycin $(25 \mu \mathrm{g} / \mathrm{ml})$ and ampicillin $(100 \mu \mathrm{g} / \mathrm{ml})$. As a nutrient-limited condition, M9 minimal medium was used to prepare total bacterial RNA in contact with vegetables.

All isogenic mutants were constructed using the $\lambda$ Red recombination system [10]. Genes constituting an operon were deleted together with other genes in the same operon. In brief, PCR products containing a kanamycin resistance gene in the middle and nucleotides identical to the flanking regions of target genes at each terminus were generated using pKD13 as a template. PCR primers used in the PCR amplification are listed in Table S2. S. Typhimurium 14028 S strain harboring the Red helper plasmid pKD46 was grown in the presence of L-arabinose $(50 \mathrm{mM})$ at $30^{\circ} \mathrm{C}$ with shaking $(220 \mathrm{rpm})$ until the $\mathrm{OD}_{600}$ reached 0.6 . Bacterial cells were then centrifuged $\left(10,000 \times g, 5 \mathrm{~min}, 4^{\circ} \mathrm{C}\right)$, resuspended in ice-cold water, and transformed by electroporation (Bio-Rad Laboratories, USA). Recombinant cells were selected by kanamycin resistance, and subjected to diagnostic PCR using the primers listed in Table S2. The kanamycin resistance cassette was removed using the FLP recombinase of PCP20.

\section{Preparation of Cabbage and Napa Cabbage}

Cabbage (Brassica oleracea var. capitata) and napa cabbage (Brassica rapa subsp. Pekinensis) were purchased from commercial markets in Suwon, Korea. To prepare vegetable samples, only leaves and stems (except roots) were used. Possible contamination with indigenous microbes was eliminated by soaking the cabbage and napa cabbage leaves and stems in water containing $0.01 \%$ sodium hypochlorite for $5 \mathrm{~min}$. Samples were washed with sterilized water twice and dried for $10 \mathrm{~min}$. After drying, the leaves and stems were cut using a knife.

\section{RNA Extraction}

To minimize RNA degradation, RNAprotect Bacteria Reagent (Qiagen, Hilden, Germany) was added to the bacterial culture, and bacterial total RNA was isolated using the RNeasy Mini Kit (Qiagen) according to the manufacturer's instructions. Isolated RNA samples were treated with Ambion Turbo DNA-free (Ambion, USA) to remove genomic DNA, and purified RNA samples were analyzed using Agilent 2100 Bioanalyzer (Agilent Technologies, USA). RNA samples with RNA integrity numbers (RIN) larger than 7 were used for RNA-sequencing analysis [11].

\section{RNA-Sequencing (RNA-Seq) and Analysis}

The rRNAs were removed using the subtractive hybridization/ bead capture system of the Ribo-Zero Kit (Epicentre Biotechnologies, USA), and an mRNA-Seq library was subsequently constructed using the Illumina TruSeq RNA Sample Preparation Kit v.2 (Illumina, USA). RNA-Seq using Illumina Hiseq generated singleend reads around $100 \mathrm{bp}$ in length. Sequencing reads were mapped to the respective genome sequences, using CLC Genomics Workbench 6.5.1 tool (CLC bio, Denmark) and all RNA-Seq data analyzed in this study were deposited in the Foodborne Pathogen Omics Research Center (http://forcdb.snu.ac.kr/data/transcriptomes). As normalization methods in RNA-Seq analysis, reads per kilobase of transcript per million mapped reads (RPKM), relative log expression (RLE), and trimmed mean of M-value (TMM) were applied [12-14]. Genes encoding proteins with similar functions were classified using Clusters of Orthologous Groups (COG) analysis [15], and heat maps were generated using Gitools v2.2.2 [16]. 


\section{Quantitative Real-Time PCR Analysis (qRT-PCR)}

Total RNA that was isolated as described above was treated with RNase-free DNase (Ambion) to remove the remaining genomic DNA. The cDNA synthesis was performed using the RNA to cDNA EcoDry Premix (Random Hexamers) (Takara, Japan). Next, qRT-PCR was performed using the StepOnePlus Real-Time PCR System (Applied Biosystems, USA) with Power SYBR Green PCR Master Mix (Applied Biosystems), and mRNA levels of each gene were normalized using those of the housekeeping gene gyrB. All primers used in this study are listed in Table S2.

\section{Biofilm Assay}

Bacterial cells cultivated in LB broth overnight were diluted into fresh $\mathrm{LB}$ broth at a 1:100 ratio and further cultured at $37^{\circ} \mathrm{C}$ for $3 \mathrm{~h}$. Bacterial cells adjusted to $\mathrm{OD}_{600} 1.0$ were mixed with LB broth at a 1:3 ratio in polystyrene and glass tubes and statically incubated at $30^{\circ} \mathrm{C}$ or $37^{\circ} \mathrm{C}$ for $48 \mathrm{~h}$ without shaking. Biofilm formation was quantified by staining with $1.0 \%$ crystal violet for $5 \mathrm{~min}$ followed by washing with phosphate buffer $(0.1 \mathrm{M}, \mathrm{pH}$ 7.4). All dye attached to the biofilm was dissolved with $33 \%$ glacial acetic acid, and $\mathrm{OD}_{595}$ was measured to quantify the total biofilm mass [17].

\section{Invasion Assay}

HeLa cells were cultivated in Dulbecco's Modified Eagle's Medium (DMEM, Corning, USA) supplemented with 10\% heatinactivated fetal bovine serum (FBS, Corning) and $50 \mu \mathrm{g} / \mathrm{ml}$ of penicillin and streptomycin. Cells were seeded onto 24-well tissue culture plates at $2 \times 10^{5}$ cells/well and incubated at $37^{\circ} \mathrm{C}$ overnight prior to bacterial infection. Bacterial cells grown in LB broth at $37^{\circ} \mathrm{C}$ overnight were washed with PBS (Corning) three times, resuspended in DMEM, and added in HeLa cells at a multiplicity of infection (MOI) of 100. Infection was initiated by centrifugation at $500 \times g$ for $5 \mathrm{~min}$, and plates were incubated for $30 \mathrm{~min}$. The cells were washed once with PBS and replenished with DMEM containing gentamicin at $100 \mu \mathrm{g} / \mathrm{ml}$ to remove extracellular bacteria. After a 2-h incubation, the cells were washed using PBS twice and lysed using 1\% Triton X-100 in PBS for 20 min. Cell lysates were serially diluted with PBS and plated on LB agar to enumerate intracellular bacteria.

\section{Motility Assay}

Bacterial cells grown in LB broth overnight were diluted into fresh LB broth and further cultured at $37^{\circ} \mathrm{C}$ for $3 \mathrm{~h}$. Bacterial strains used in the motility assay did not show any growth differences compared to that of the wild-type strain, and bacterial cultures were adjusted to an $\mathrm{OD}_{600}$ of 1.0. Next, $2 \mu \mathrm{l}$ of bacterial culture was injected onto LB agar containing $0.3 \%$ agar. LB agar plates were statically incubated at $30^{\circ} \mathrm{C}$ or $37^{\circ} \mathrm{C}$ for $5 \mathrm{~h}$, and the diameters of the bacterial growth halos were measured. Motility was measured in at least three independent experiments, and then the halo diameters were averaged.

\section{Reactive Oxygen Species (ROS) Resistance Test}

Bacterial cells were cultured in LB broth overnight and diluted to fresh LB broth. The cells were next cultured for $3 \mathrm{~h}$, washed using PBS, and adjusted to $2 \times 10^{7}$ cells $/ \mathrm{ml}$ in PBS. Next, the cells were treated with hydrogen peroxide at 1 to $4 \mathrm{mM}$ for $15 \mathrm{~min}$ before being immediately diluted with PBS and spotted onto LB agar plates or spread on agar plates for enumeration. Bacterial counting was performed at least three times and the numbers were averaged.

\section{Statistical Analysis}

The data were presented as the mean with standard deviation from at least three independent tests and analyzed using the Student's $t$-test. A $p$ value of 0.05 or less was regarded as being statistically significant.

\section{Results}

Effect of Cabbage and Napa Cabbage on Growth of Salmonella

Some foodborne pathogens including E. coli and Salmonella
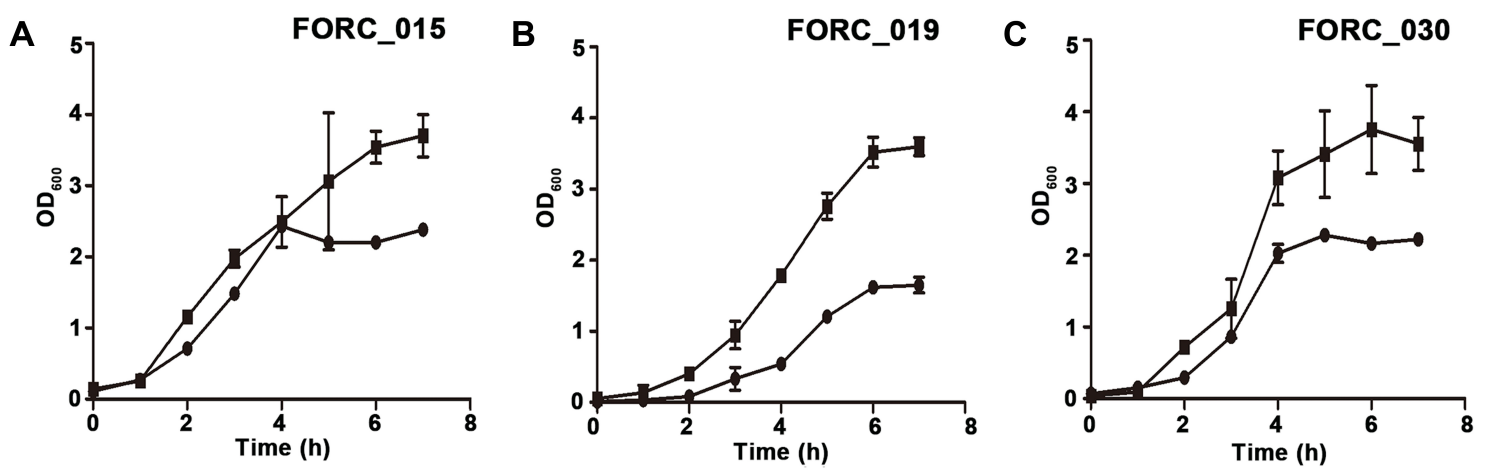

Fig. 1. Growth curves of bacteria in contact with vegetables.

Three FORC strains of FORC_015 (A), FORC_019 (B), and FORC_030 (C) were cultured in M9 minimal medium broth supplemented with cabbage (A, C) or napa cabbage (B). Growth without vegetables (circle) or with vegetables (square) was measured every hour. 
have the ability to survive in secondary habitats such as vegetables, and their colonization of vegetable surfaces was found to be accelerated when vegetable tissues were macerated, leading to leakage of cellular components [18]. In order to analyze the Salmonella transcriptome when in contact with vegetables, Salmonella strains FORC_015, FORC_019, and FORC_030 were cultivated in M9 minimal medium broth supplemented with cut cabbage and napa cabbage, and total bacterial RNAs were isolated at around $3 \mathrm{~h}$ when cells reached the mid-logarithmic growth phase. As observed in other cases, Salmonella replicated faster when the vegetable samples were provided as additional nutrients (Fig. 1).

Comprehensive Transcriptomic Analysis of Salmonella enterica spp. upon Contact with Cabbage and Napa Cabbage

The Salmonella transcriptome was analyzed in the presence and absence of cabbage or napa cabbage, and the genes with changes in expression by two-fold or more upon contact with vegetables were sorted ( $p$ value $<0.05$ ). To delineate the physiological changes that occurred in the bacterial cells during contact with vegetables, the differentially expressed genes (DEGs) were categorized based on their predicted functions using COG designations [15]. In FORC_015, FORC_019, and FORC_030 co-cultured with cabbage, $9.30 \%(428 / 4601), 5.01 \%(242 / 4519)$; and $8.29 \%(400 / 4929)$ of the genes had significantly changed expressions.

Functional grouping of DEGs varied among the three FORC strains, indicating strain-dependent differences in transcriptional responses to vegetables. However, in all three bacterial strains, co-cultivation with vegetables led to expression changes in genes associated with energy production and conversion (16.55\% in FORC_015, 3.11\% in FORC_019, and 6.92\% in FORC_030), amino acid transport and metabolism (11.94\% in FORC_015, 6.92\% in FORC_019, and $7.63 \%$ in FORC_030), and carbohydrate transport and metabolism (6.45\% in FORC_015, 10.89\% in FORC_019, and $3.69 \%$ in FORC_030) (Fig. 2). Furthermore, the three FORC
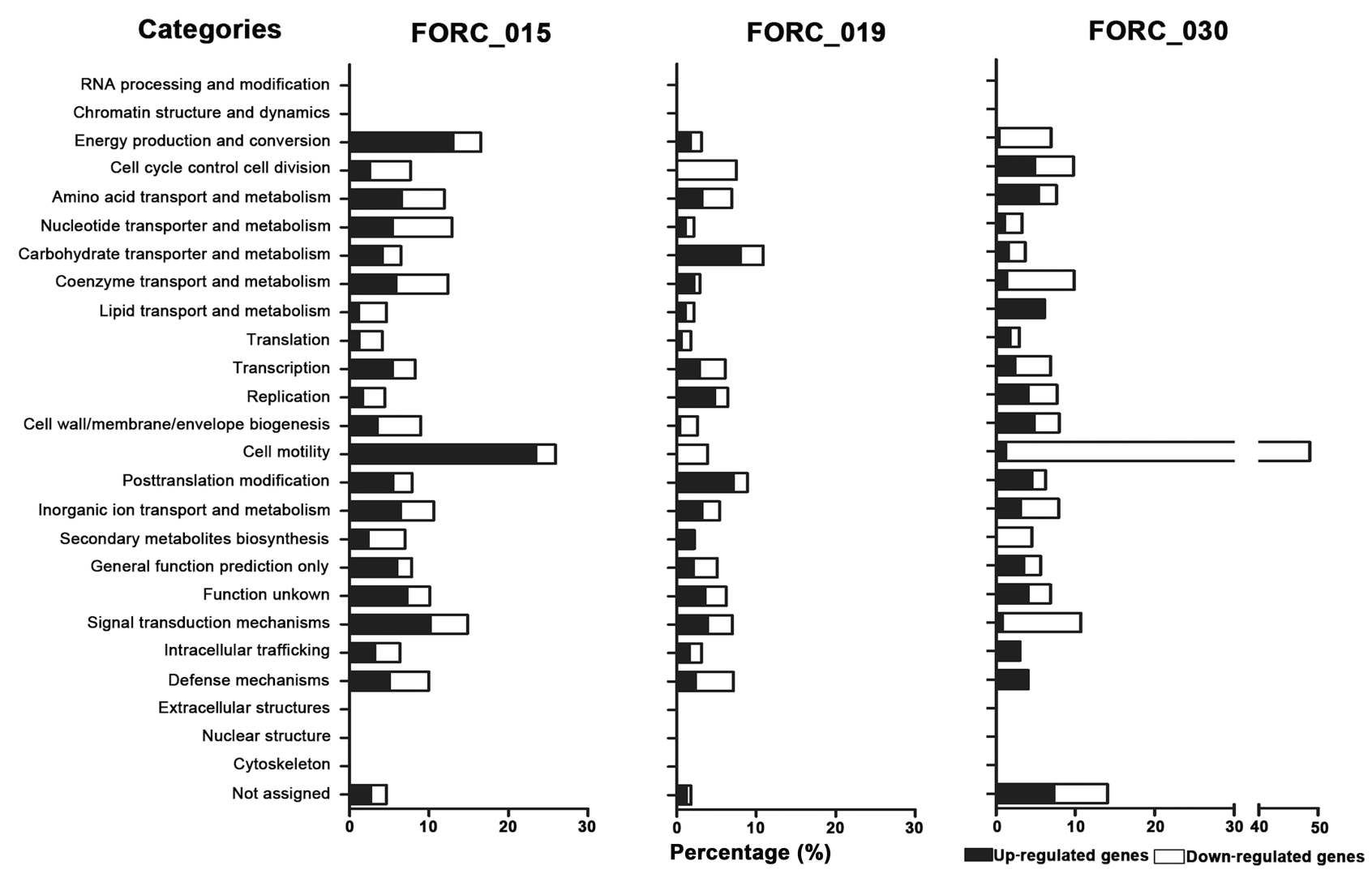

Fig. 2. Functional categorization of differentially regulated genes (DEGs).

Functional groups of genes that showed significant expression changes upon contact with vegetables were graphically compared, based on COG analysis. Genes up- or down-regulated by more than 2-fold in the presence of vegetables were sorted in each COG group, and their percentages are indicated by black and white bars, respectively. 
strains showed significant changes in the expression of genes related to cell motility (25.93\% in FORC_015, 3.90\% in FORC_019, and $58.54 \%$ in FORC_030) and signal transduction mechanisms $(14.84 \%$ in FORC_015, 6.98\% in FORC_019, and 10.69\% in FORC_030) upon the contact with vegetables (Fig. 2). These results suggested that Salmonella might sense various nutrients released from vegetable tissues and take advantage of them for proliferation, which is accordant with the increased Salmonella growth in the presence of vegetables (Fig. 1).

\section{Differential Regulation of Salmonella Virulence Determinants in Response to Cabbage and Napa Cabbage}

Despite the intimate association between vegetables and Salmonella, the underlying mechanism by which Salmonella persists on the surface of vegetables or in their cellular compartments has not been investigated in detail. In an effort to understand how the expression of virulence genes upon contact with raw vegetable is coordinated in Salmonella, the transcriptional changes in representative virulence determinants were evaluated. In animal models of Salmonella infection, Salmonella generally employs two different type III secretion systems (T3SSs) produced from Salmonella pathogenicity island (SPI)-1 and SPI-2 as primary virulence determinants. Virulence factors translocated via SPI-1 T3SS exert their roles during Salmonella invasion into host cells, while those delivered via SPI-2 T3SS are required for bacterial survival and proliferation inside host cells [19, 20]. The possibility that Salmonella exploits plant tissues and cells as secondary reservoirs in unfavorable environments was examined by comparing the expressions of SPI-1 and SPI-2 genes in the presence and absence of vegetables. SPI-1 genes were up-regulated in FORC_019 and FORC_030 in response to napa cabbage and cabbage, respectively, while SPI-2 genes were moderately down-regulated in all three strains (Fig. 3). The increased SPI-1 expression when in contact with vegetables suggests that Salmonella may utilize SPI-1 T3SS during internalization into plant cells, similar to the mechanism used in animal cells. Recent studies support this possibility, showing that Salmonella effectors delivered through SPI-1 T3SS are required for Salmonella to dampen the plant immune system and colonize plant tissues [21, 22].

\section{Defining Genes Differentially Expressed in Contact with Cabbage and Napa Cabbage}

To further understand the strategy employed by Salmonella for adaptation to vegetable environments, genes up- or down-regulated by more than 2-fold in at least two Salmonella strains were sorted (Table S3). The roles of DEGs might be associated with bacterial adaptation to not only plants but also other hostile niches such as animal hosts. Nontyphoidal Salmonella serovars used in this study, including Typhimurium, Enteritidis, and Mbandaka, are important foodborne pathogens that cause gastroenteritis,
SPI-1

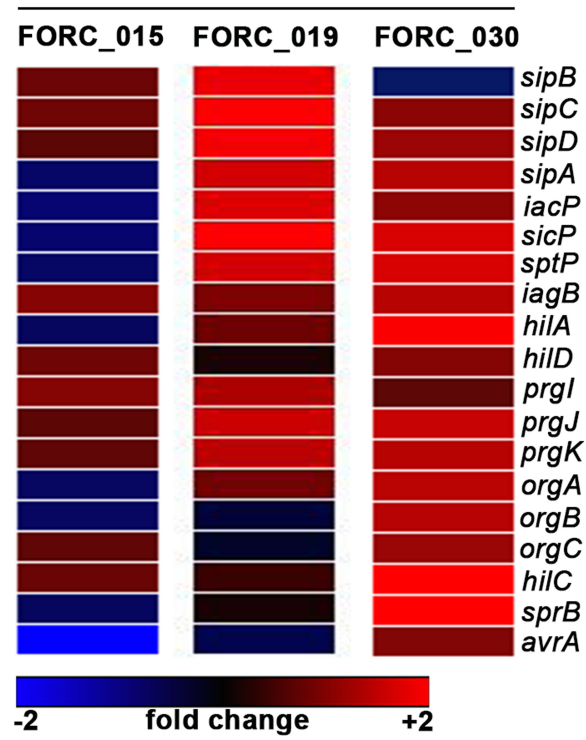

SPI-2

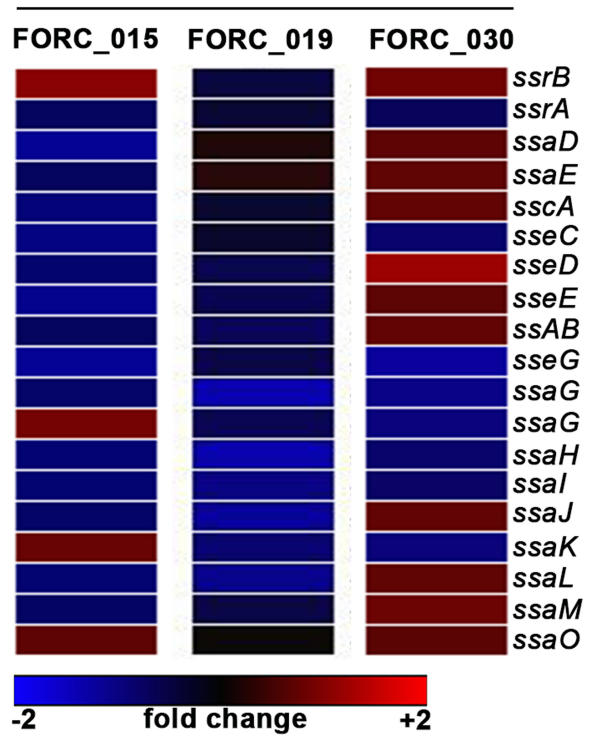

Fig. 3. Expression of SPI-1 and SPI-2 genes in three FORC strains in response to vegetables.

Heat maps of genes associated with SPI-1 and SPI-2 were represented using fold-changes $\left(\log _{2}\left[\mathrm{RPKM}_{\text {Vegetable }} / \mathrm{RPKM}_{\text {Control }}\right]\right)$ and depicted using a colorimetric gradient: down-regulation in blue and up-regulation in red. 


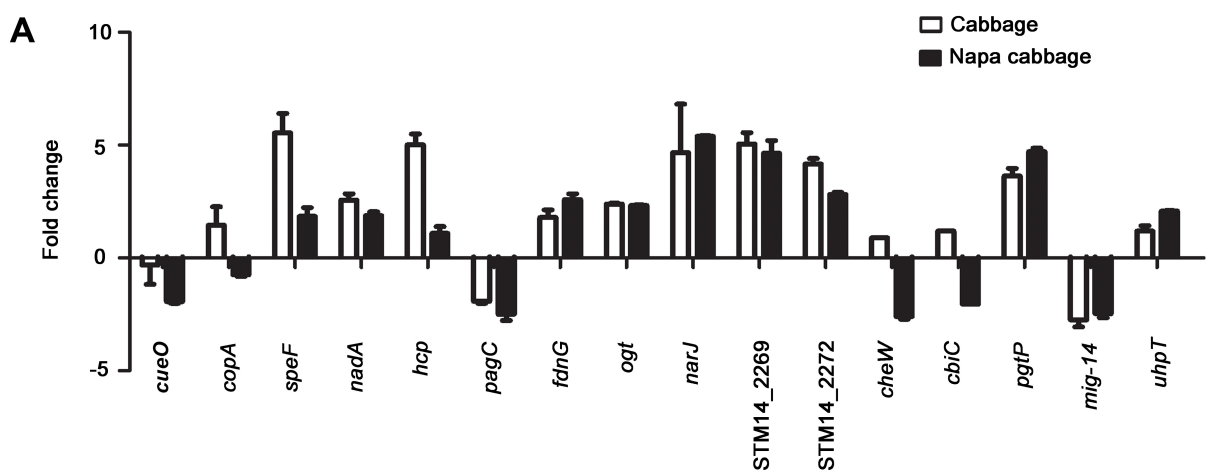

B

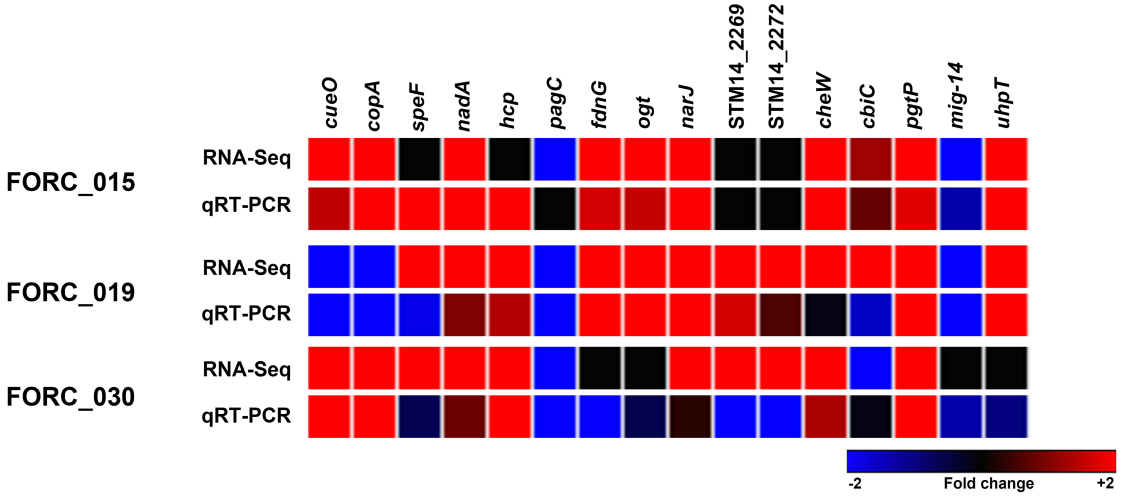

Fig. 4. Validation of differentially regulated genes (DEGs) in S. Typhimurium 14028 in contact with cabbage and napa cabbage. For 16 DEGs, qRT-PCR was performed in S. Typhimurium 14028 S in response to cabbage (white bar) and napa cabbage (black bar) (A). Foldchanges in $\log _{2}\left[\mathrm{Ct}_{\text {vegetable }} / \mathrm{Ct}_{\text {Control }}\right]$ were obtained from three independent experiments, and the averages are plotted with their standard deviations. The expressions of 16 DEGs were examined in the three FORC strains using qRT-PCR (B). The qRT-PCR fold-changes in $\log _{2}\left[\mathrm{Ct}_{\text {Vegetable }} / \mathrm{Ct}_{\text {Control }}\right]$ were depicted using a colorimetric gradient heat map, in parallel with RNA-Seq fold-changes in $\left(\log _{2}\left[R P K M_{V e g e t a b l e} / R P K M_{\text {Control }}\right]\right)$ for each strain: down-regulation in blue and up-regulation in red.

bacteremia, and subsequent focal infection. However, most molecular studies have been performed using several typical S. Typhimurium strains, such as 14028 S. In an effort to comprehensively decipher the bacterial transcriptional regulation at the molecular level, the expression levels of DEGs were evaluated in S. Typhimurium 14028S, a representative virulent strain extensively used in molecular studies. Among the 51 DEGs identified in the transcriptomic analyses, 16 genes showed significant changes in their expression in S. Typhimurium 14028S in contact with cabbage or napa cabbage (Fig. 4A), while the transcription levels of other genes were not altered in response to vegetables. When the expression of $16 \mathrm{DEGs}$ was examined in the three FORC strains using qRT-PCR, some genes such as speF and cbiC showed a discrepancy in their expression between RNA-Seq and qRT-PCR results (Fig. 4B), indicating the importance of RNA-Seq validation using other approaches such as qRT-PCR. Expression changes of some genes in the three FORC isolates were not represented in
S. Typhimurium 14028S (compare Figs. 4A and 4B), suggesting differential transcriptional regulation between Salmonella strains. In spite of the discordant gene expressions among the bacterial strains, these 16 genes with significant transcriptional alterations in S. Typhimurium 14028S deserve to be further tested as growth or survival determinants important for Salmonella adaptation and survival under unfavorable environments such as those presented by plants and animals.

\section{Exploring Genes Associated with Salmonella Adaption into Hostile Environments}

To determine the genetic loci required for Salmonella adaptation to vegetable environments, the 16 DEGs were deleted individually in the chromosome of $S$. Typhimurium $14028 \mathrm{~S}$ as described in Materials and Methods, and the growth of each mutant strain was measured with or without vegetables. All mutant strains except $\Delta$ nadA showed comparable growth rates with that of the wild-type strain 

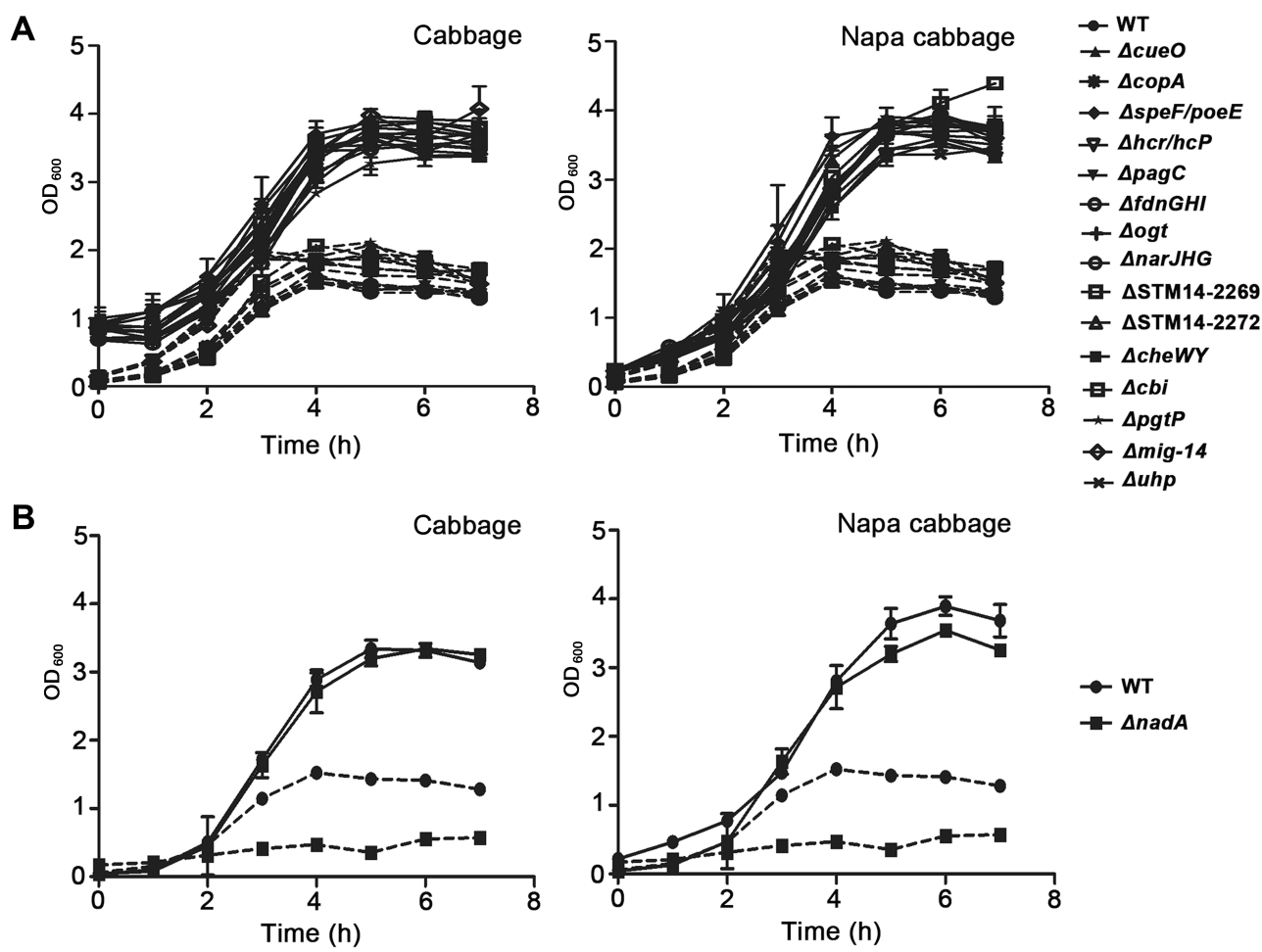

Fig. 5. Growth curves of Salmonella mutants lacking 16 differentially regulated genes (DEGs).

S. Typhimurium 14028 S lacking each DEG was cultured with (solid line) or without (dotted line) cabbage and napa cabbage. All 15 mutant strains except $\Delta$ nadA showed comparable growth rates to that of the wild-type Salmonella strain (A). The $\Delta$ nadA mutant showed growth defects in the absence of vegetables, but its growth was rescued with the addition of vegetables (B).

regardless of the presence of cabbage or napa cabbage (Fig. 5A). However, the mutant lacking nadA had attenuated growth in M9 minimal medium (Fig. 5B), whereas its growth was not attenuated in LB medium when compared to other mutant strains (Fig. S1). The gene nadA encodes quinolinate synthase, which is required for the biosynthesis of nicotinamide adenine dinucleotide (NAD). Interestingly, the supplementation with cabbage or napa cabbage complemented the growth attenuation of $\Delta$ nadA strain (Fig. 5B), suggesting that bioactive compounds released from vegetable tissues enabled the mutant to circumvent the de novo NAD biosynthesis pathway.

In an effort to define new virulence determinants associated with Salmonella adaptation and survival under unfavorable environments, we next examined the ability for the bacterial cells to invade into animal cells in the absence of the 16 DEGs. The invasion ability of Salmonella lacking speF/potE (STM14_0818 and STM14_0817), fdnGHI (STM14_1894 to STM14_1892), ogt (STM14_2006), STM14_2269, and the $c b i$ operon (STM14_2513 to STM14_2523) into epithelial cells was attenuated, indicating positive roles of these DEGs in promoting Salmonella invasion (Fig. 6). The genes speF and
potE encode ornithine decarboxylase and putrescine transporter, respectively, and are both associated with homeostasis of putrescine, a small-cationic polyamine required for Salmonella resistance and virulence during host infection [23]. The operon $f d n G H I$ encodes formate dehydrogenase $\mathrm{N}$, which is required for the oxidation of formate to carbon dioxide, donating electrons to the quinone pool for the reduction of anaerobic respiratory substrates [24]. The ogt gene encodes methylated DNA protein cysteine methyltransferase, which repairs alkylated DNA, and is known to be required for bacterial resistance to DNA mutations caused by alkylating agents [25]. STM14_2269 has been postulated to encode a lytic enzyme, and the $c b i$ operon encodes multiple enzymes for the biosynthesis of cobalamin (vitamin $B_{12}$ ) [26]. The invasion assay results suggest that these five genetic loci might be novel virulence determinants associated with Salmonella adaption to host animals.

\section{Understanding Roles of $c b i$ Operon as a Virulence Determinant in Salmonella}

Among the five genetic loci required for Salmonella to 


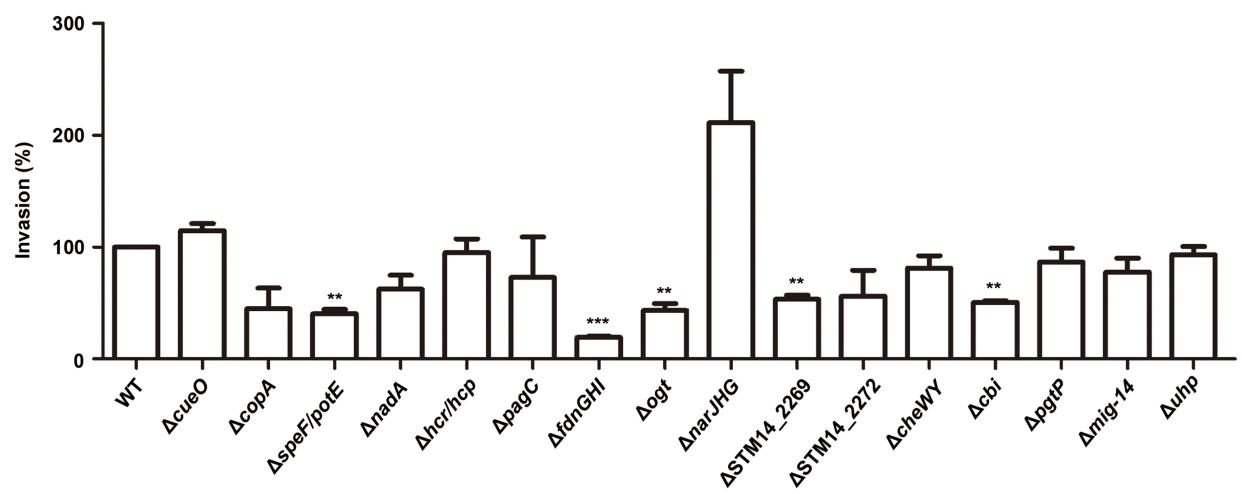

Fig. 6. Invasion assay of Salmonella strains lacking 16 differentially regulated genes (DEGs).

HeLa cells were infected with 16 S. Typhimurium 14028 S derivatives lacking each DEG in parallel with wild-type strain at a multiplicity of infection of 100. Intracellular bacteria were enumerated at $2.5 \mathrm{~h}$ post-infection, and the bacterial numbers relative to that of the wild-type strain were plotted as the average of three independent experiments $\left(* *, p\right.$ value $<0.05 ;{ }^{* * *}, p$ value $\left.<0.005\right)$.

invade cells, we further investigated the roles of the $c b i$ operon as a virulence determinant. Cobalamin is known to function as a cofactor in diverse reactions in $S$. Typhimurium, including methyltransferase-mediated methionine biosynthesis, degradation of ethanolamine to acetaldehyde, and propanediol dehydratase-mediated propanediol degradation [27]. In view of the multifaceted roles of cobalamin in Salmonella, we characterized the physiological features of the $\Delta c b i$ mutant strain, which might be associated with its attenuated virulence.

Bacterial motility in the absence of the $c b i$ operon was evaluated since motility is required for evading stressful stimuli as well as internalization into host cells. Salmonella lacking the $c b i$ operon showed attenuated motility on agar plates (Fig. 7A), suggesting that the decrease in its ability to invade cells might be attributed to the attenuated motility toward host cells at least in part.

In addition to motility, the invasion ability of Salmonella is influenced by multiple virulence factors. Among these, SPI-1 is a prominent requirement for Salmonella invasion. However, a lack of the cbi operon did not influence the transcriptional expression of SPI-1 genes, which encode a component of the SPI-1 T3SS apparatus (InvA) and its secretion substrate (SipC), suggesting that the decreased invasion ability of the $\Delta c b i$ strain was not caused by malfunctioning SPI-1 T3SS (Fig. 7B). Interestingly, the lack of $c b i$ operon increased the transcription of $p d u F$ and $p d u A$ genes, which compose $p d u$ operon (Fig. 7B). The role of $p d u$ operon in Salmonella physiology and virulence has been recently addressed as described in Discussion.

We next investigated the role of the $c b i$ operon in Salmonella resistance under stressful environments. The altered expression of $c b i$ operon in contact with vegetables might be associated with bacterial resistance against unfavorable stimuli encountered during the adaptation to plant environments. When bacteria are exposed to hostile conditions such as nutrient starvation as well as physical and chemical stressors, they form multicellular complexes called biofilms, which provide a shelter for the bacterial cells to cope with transient or permanent stress conditions [28]. Salmonella lacking the cbi operon developed biofilms better than the wild-type strain on the surface of polystyrene, but showed no difference in biofilm formation on the surface of glass (Fig. 7C). This result showed that the ability to form biofilms was altered depending on the surface materials with different physical properties.

A plausible stressor encountered during bacterial adaptation to plant tissues is oxidative stress. One of the earliest and best-characterized responses of plant-to-pathogen invasion is the oxidative burst. High concentrations of ROS are produced at the plasma membrane in the vicinity of the pathogen $[29,30]$. Therefore, bacterial resistance against hydrogen peroxide was compared between wild-type and $\Delta c b i$ strains. However, the two strains showed comparable resistance against hydrogen peroxide, indicating no relevance of the $c b i$ operon in ROS tolerance (Fig. 7D).

\section{Discussion}

In recent decades, an increasing number of surveys and studies have noticed increased risks of produce contaminated with enteric pathogens. According to the United States outbreak data from the 1970s to the 1990s, the proportion of foodborne outbreaks attributable to produce increased 
A
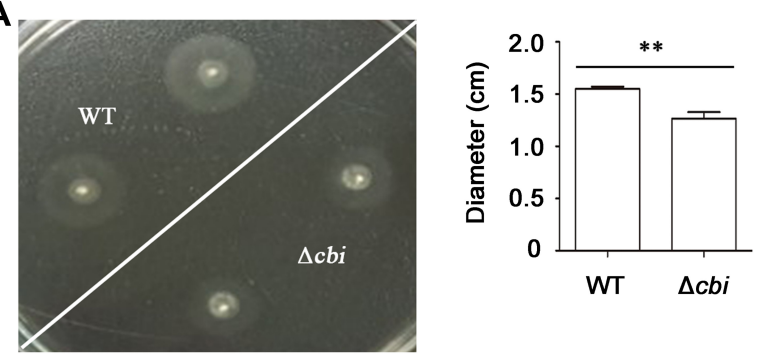

C

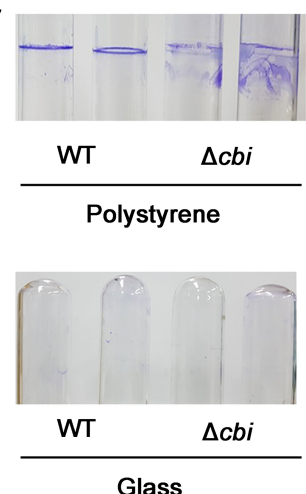

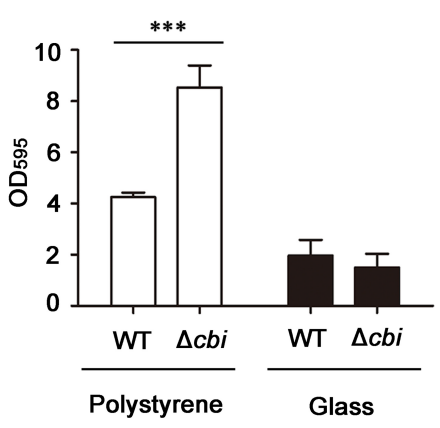

B

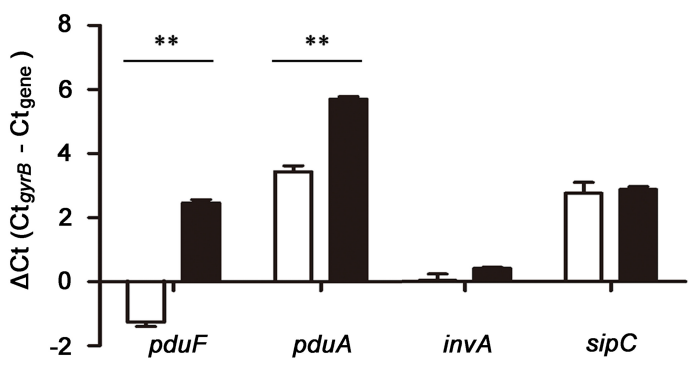

D

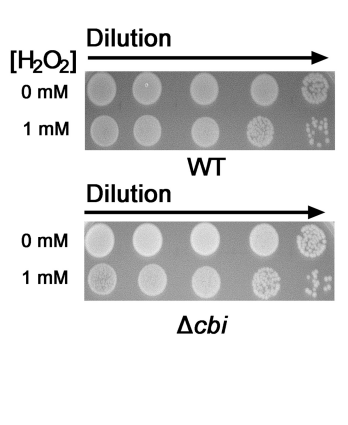

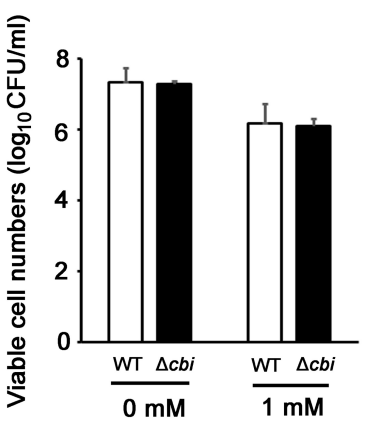

Fig. 7. Physiological characterization of $\Delta c b i$ mutant.

(A) Motility was compared between wild-type and $\Delta c b i$ mutant strains, and the diameters were averaged and plotted from three independent experiments $\left({ }^{*}, p\right.$ value $\left.<0.05\right)$. (B) Expressions of two $p d u$ operon genes and two SPI-1 genes were compared between the wild-type (white bar) and $\Delta c b i$ mutant (black bar) strains using qRT-PCR. Ct values of target genes were normalized using that of the constitutive gene gyrB in each strain. The graph was produced using three independent assays $\left({ }^{* *}, p\right.$ value $\left.<0.05\right)$. (C) Biofilm formation was tested in polystyrene and glass tubes. The biofilm stained using crystal violet solution was measured at an optical density of $595 \mathrm{~nm}$ and plotted using results of three tests ${ }^{* * *}, p$ value $<0.005$ ). (D) Bacterial resistance against hydrogen peroxide was compared between the wild-type and $\Delta c b i$ mutant strains. Bacterial cells treated with hydrogen peroxide for $15 \mathrm{~min}$ were serially diluted and spotted or spread onto LB agar plates. The result is a representative image of three independent experiments.

from $0.7 \%$ to $6 \%$ [31]. More recently, between 1990 and 2005, produce has been associated with $13 \%$ of the identified food source outbreaks [32]. Salmonella enterica is a major species that accounts for a large proportion of foodborne outbreaks associated with the consumption of fresh produce. S. enterica spp. are able to colonize green leafy vegetables such as cabbage and lettuce [9], and survive more than 100 days without provoking disease symptoms in plants [33, 34]. Although the overall populations of inoculated pathogens tend to decline, the persistence of as low as a few cells might still cause serious outbreaks [35]. The risk of Salmonella internalization into diverse edible tissue poses a threat to food processing environments and public health [36].

In this study, three Salmonella spp. strains were incubated with cabbage or napa cabbage tissues, and their transcriptome profiles were acquired to understand how
Salmonella adapts to unfavorable plant environments. Genes up- or down-regulated upon contact with plant tissues were sorted, and their roles were evaluated in the $S$. Typhimurium $14028 \mathrm{~S}$ strain to determine genes implicated in bacterial survival and virulence under unfavorable environments. The $S$. Typhimurium 14028 S strain is a representative strain widely used in virulence studies and provides extensive information at the molecular level. Among the 16 genes with significant expression changes in $S$. Typhimurium $14028 \mathrm{~S}$ in response to cabbage and napa cabbage, nadA was required for bacterial growth under nutrient-restricted environments (Fig. 5B), while speF/potE, $f d n G H I$, ogt, STM14_2269, and the cbi operon were implicated in the ability of Salmonella to invade host epithelial cells (Fig. 6). These genes are presumably virulence determinants associated with Salmonella virulence and survival. In this context, diverse control measures inactivating the roles of 
these genes can be developed to control Salmonella contamination and infection in the food industry.

Quinolinate synthase encoded by nadA catalyzes the condensation reaction between 2-iminosuccinate and glycerone phosphate, resulting in quinolinate formation in the de novo NAD biosynthesis pathway starting from aspartate. NAD participates in varied metabolic pathways as a donor of ADP-ribose moieties in ADP-ribosylation reactions, a precursor of the secondary messenger molecule cyclic ADP-ribose, or a substrate for bacterial DNA ligases [37]. Therefore, the lack of $\operatorname{nadA}$ can dampen the biosynthesis of NAD from amino acids in cells, leading to a malfunction of multiple metabolic pathways. We observed that the attenuated growth of the $\Delta$ nadA strain was complemented by the addition of cabbage or napa cabbage (Fig. 5B). As an alternative to de novo biosynthesis for maintaining cellular NAD, bacteria recycle pyridine compounds including nicotinic acid and nicotinamide that are scavenged from the environment [38]. Cabbage and napa cabbage are rich sources of minerals and vitamins, including magnesium, calcium, iron, vitamin $\mathrm{A}$, vitamin $\mathrm{C}$, and vitamin $B$ complexes. In particular, niacin (vitamin $B_{3}$; nicotinic acid) is abundant; at $0.25 \mathrm{mg}$ and $0.5 \mathrm{mg}$ per $100 \mathrm{~g}$ cabbage and napa cabbage, respectively [39]. Therefore, the $\triangle$ nadA strain was likely to scavenge niacin from the vegetable exudates and circumvent the compromised de novo biosynthesis pathway. This phenomenon indicated that inactivation of NAD homeostasis can be a promising control strategy against Salmonella proliferation.

As another important factor required for Salmonella virulence, the $c o b$ operon showed significant expression changes upon contact with cabbage and napa cabbage among the 4 different Salmonella strains. The cob operon, which consists of vitamin $B_{12}$ biosynthetic genes, is induced in response to 1,2-propanediol and is co-regulated with the $p d u$ operon. The $p d u$ operon encodes a polyhedral body and its enzyme components, which degrade 1,2-propanediol as carbon and energy sources in the presence of a cofactor vitamin $B_{12}$ [40]. Furthermore, 1,2- propanediol, the endproduct of bacterial fermentation from L-fucose or Lrhamnose, is an abundant metabolite present in the intestinal lumen [41]. Therefore, Salmonella with the ability to utilize 1,2-propanediol outcompetes the gut microbiota $[42,43]$. A lack of the $c b i$ operon increased the expression of $p d u$ genes (Fig. 7), suggesting that the $\Delta c b i$ strain might outcompete the commensal bacteria in the intestinal lumen when administered into host animals. With respect to the additional role of the $p d u$ operon in Salmonella, propionylCoA, the intermediate of 1,2-propanediol utilization pathway, was shown to reduce the protein stability of HilD, a Salmonella SPI-1 transcriptional regulator, which in turn attenuated Salmonella invasion into intestinal epithelial cells [44]. However, a lack of the $c b i$ operon did not influence the expressions of SPI- 1 genes, indicating that the increase in $p d u$ transcription in the $\Delta c b i$ strain did not lead to the down-regulation of SPI-1 via propionyl-CoA (Fig. 7).

In conclusion, the transcriptomes of Salmonella spp. strains in contact with cabbage and napa cabbage provided insights into the overall bacterial transcriptional response to vegetables. The comprehensive transcriptomes help us better understand the bacterial adaption strategies in vegetables as well as devise control measures to inactivate essential bacterial determinants required for Salmonella proliferation in diverse conditions, such as soil and produce environments.

\section{Conflict of Interest}

The authors have no financial conflicts of interest to declare.

\section{Acknowledgements}

This research was supported by the Ministry of Food and Drug safety (14162MFDS972), and the Basic Science Research Program through the National Research Foundation of Korea (NRF) funded by the Ministry of Science, ICT \& Future Planning (NRF- 2018R1A2B6007304).

\section{References}

1. Gal-Mor O, Boyle EC, Grassl GA. 2014. Same species, different diseases: how and why typhoidal and non-typhoidal Salmonella enterica serovars differ. Front Microbiol. 5: 391.

2. Yaron S, Romling U. 2014. Biofilm formation by enteric pathogens and its role in plant colonization and persistence. Microb. Biotechnol. 7: 496-516.

3. Ochman H, Groisman EA. 1995. The evolution of invasion by enteric bacteria. Can. J. Microbiol. 41: 555-561.

4. Fukuyama S, Watanabe Y, Kondo N, Nishinomiya T, Kawamoto S, Isshiki K, et al. 2009. Efficiency of sodium hypochlorite and calcinated calcium in killing Escherichia coli O157:H7, Salmonella spp., and Staphylococcus aureus attached to freshly shredded cabbage. Biosci. Biotechnol. Biochem. 73: 9-14.

5. Shirron N, Kisluk G, Zelikovich Y, Eivin I, Shimoni E, Yaron S. 2009. A comparative study assaying commonly used sanitizers for antimicrobial activity against indicator 
bacteria and a Salmonella Typhimurium strain on fresh produce. J. Food Prot. 72: 2413-2417.

6. Lockhart WR, Holt JG. 1964. Numerical classification of Salmonella serotypes. J. Gen. Microbiol. 35: 115-124.

7. Jackson BR, Griffin PM, Cole D, Walsh KA, Chai SJ. 2013. Outbreak-associated Salmonella enterica serotypes and food Commodities, United States, 1998-2008. Emerg. Infect. Dis. 19: $1239-1244$.

8. Herman KM, Hall AJ, Gould LH. 2015. Outbreaks attributed to fresh leafy vegetables, United States, 1973-2012. Epidemio Infect. 143: 3011-3021.

9. Kroupitski Y, Golberg D, Belausov E, Pinto R, Swartzberg D, Granot D, et al. 2009. Internalization of Salmonella enterica in leaves is induced by light and involves chemotaxis and penetration through open stomata. Appl. Environ. Microbiol. 75: 6076-6086.

10. Datsenko KA, Wanner BL. 2000. One-step inactivation of chromosomal genes in Escherichia coli K-12 using PCR products. Proc. Natl. Acad. Sci. USA 97: 6640-6645.

11. Schroeder A, Mueller O, Stocker S, Salowsky R, Leiber M, Gassmann M, et al. 2006. The RIN: an RNA integrity number for assigning integrity values to RNA measurements. BMC Mol. Biol. 7: 3.

12. Robinson MD, Oshlack A. 2010. A scaling normalization method for differential expression analysis of RNA-seq data. Genome Biol. 11: R25.

13. Dillies MA, Rau A, Aubert J, Hennequet-Antier C, Jeanmougin M, Servant N, et al. 2013. A comprehensive evaluation of normalization methods for Illumina highthroughput RNA sequencing data analysis. Brief Bioinform. 14: 671-683.

14. Risso D, Ngai J, Speed TP, Dudoit S. 2014. Normalization of RNA-seq data using factor analysis of control genes or samples. Nat. Biotechnol. 32: 896-902.

15. Tatusov RL, Koonin EV, Lipman DJ. 1997. A genomic perspective on protein families. Science 278: 631-637.

16. Perez-Llamas C, Lopez-Bigas N. 2011. Gitools: analysis and visualisation of genomic data using interactive heat-maps. PLoS One 6: e19541.

17. Vikram A, Jesudhasan PR, Jayaprakasha GK, Pillai BS, Patil BS. 2010. Grapefruit bioactive limonoids modulate E. coli O157:H7 TTSS and biofilm. Int. J. Food Microbiol. 140: 109-116.

18. Cooley M, Carychao D, Crawford-Miksza L, Jay MT, Myers C, Rose C, et al. 2007. Incidence and tracking of Escherichia coli O157:H7 in a major produce production region in California. PLoS One 2: e1159.

19. Galan JE. 2001. Salmonella interactions with host cells: type III secretion at work. Annu. Rev. Cell Dev. Biol. 17: 53-86.

20. McGhie EJ, Brawn LC, Hume PJ, Humphreys D, Koronakis V. 2009. Salmonella takes control: effector-driven manipulation of the host. Currt. Opin. Microbiol. 12: 117-124.

21. Schikora A, Virlogeux-Payant I, Bueso E, Garcia AV, Nilau T,
Charrier A, et al. 2011. Conservation of Salmonella infection mechanisms in plants and animals. PLoS One 6: e24112.

22. Shirron N, Yaron S. 2011. Active suppression of early immune response in tobacco by the human pathogen Salmonella Typhimurium. PLoS One 6: e18855.

23. Espinel IC, Guerra PR, Jelsbak L. 2016. Multiple roles of putrescine and spermidine in stress resistance and virulence of Salmonella enterica serovar Typhimurium. Microb. Pathog. 95: 117-123.

24. Jormakka M, Tornroth S, Byrne B, Iwata S. 2002. Molecular basis of proton motive force generation: structure of formate dehydrogenase-N. Science 295: 1863-1868.

25. Mackay WJ, Han S, Samson LD. 1994. DNA alkylation repair limits spontaneous base substitution mutations in Escherichia coli. J. Bacteriol. 176: 3224-3230.

26. Roessner CA, Warren MJ, Santander PJ, Atshaves BP, Ozaki S, Stolowich NJ, et al. 1992. Expression of 9 Salmonella typhimurium enzymes for cobinamide synthesis. Identification of the 11-methyl and 20-methyl transferases of corrin biosynthesis. FEBS Lett. 301: 73-78.

27. Paiva JB, Penha Filho RA, Junior AB, Lemos MV. 2011. Requirement for cobalamin by Salmonella enterica serovars Typhimurium, Pullorum, Gallinarum and Enteritidis during infection in chickens. Braz. J. Microbiol. 42: 1409-1418.

28. Van Houdt R, Michiels CW. 2010. Biofilm formation and the food industry, a focus on the bacterial outer surface. J. Appl. Microbiol. 109: 1117-1131.

29. Wojtaszek P. 1997. Oxidative burst: an early plant response to pathogen infection. Biochem. J. 322 (Pt 3): 681-692.

30. Lamb C, Dixon RA. 1997. The oxidative burst in plant disease resistance. Annu. Rev. Plant Physiol. Plant Mol. Biol. 48: 251-275.

31. Sivapalasingam S, Friedman CR, Cohen L, Tauxe RV. 2004. Fresh produce: a growing cause of outbreaks of foodborne illness in the United States, 1973 through 1997. J. Food Prot. 67: 2342-2353.

32. Olaimat AN, Holley RA. 2012. Factors influencing the microbial safety of fresh produce: a review. Food Microbiol. 32: 1-19.

33. Kisluk G, Yaron S. 2012. Presence and persistence of Salmonella enterica serotype typhimurium in the phyllosphere and rhizosphere of spray-irrigated parsley. Appl. Environ. Microbiol. 78: 4030-4036.

34. Markland SM, Shortlidge KL, Hoover DG, Yaron S, Patel J, Singh A, et al. 2013. Survival of pathogenic Escherichia coli on basil, lettuce, and spinach. Zoonoses Public Health 60: 563-571.

35. Douesnard-Malo F, Daigle F. 2011. Increased persistence of Salmonella enterica serovar Typhi in the presence of Acanthamoeba castellanii. Appl. Environ. Microbiol. 77: 76407646.

36. Lieberman VM, Zhao IY, Schaffner DW, Danyluk MD, Harris LJ. 2015. Survival or growth of inoculated Escherichia coli O157:H7 and Salmonella on yellow onions (Allium cepa) 
under conditions simulating food service and consumer handling and storage. J. Food Prot. 78: 42-50.

37. Foster JW, Park YK, Penfound T, Fenger T, Spector MP. 1990. Regulation of NAD metabolism in Salmonella typhimurium: molecular sequence analysis of the bifunctional nadR regulator and the nadA-pnuC operon. J. Bacteriol. 172: 4187-4196.

38. Foster JW, Moat AG. 1980. Nicotinamide adenine dinucleotide biosynthesis and pyridine nucleotide cycle metabolism in microbial systems. Microbiol. Rev. 44: 83-105.

39. Baianova Iu I, Trubachev IN. 1981. [Comparative evaluation of the vitamin composition of unicellular algae and higher plants grown under artificial conditions]. Prikl. Biokhim. Mikrobiol. 17: 400-407.

40. Obradors N, Badia J, Baldoma L, Aguilar J. 1988. Anaerobic metabolism of the L-rhamnose fermentation product 1,2propanediol in Salmonella typhimurium. J. Bacteriol. 170:
2159-2162.

41. Boronat A, Aguilar J. 1981. Metabolism of L-fucose and L-rhamnose in Escherichia coli: differences in induction of propanediol oxidoreductase. J. Bacteriol. 147: 181-185.

42. Lawley TD, Bouley DM, Hoy YE, Gerke C, Relman DA, Monack DM. 2008. Host transmission of Salmonella enterica serovar Typhimurium is controlled by virulence factors and indigenous intestinal microbiota. Infect. Immun. 76: 403-416.

43. Stecher B, Robbiani R, Walker AW, Westendorf AM, Barthel M, Kremer M, et al. 2007. Salmonella enterica serovar typhimurium exploits inflammation to compete with the intestinal microbiota. PLoS Biol. 5: 2177-2189.

44. Hung CC, Garner CD, Slauch JM, Dwyer ZW, Lawhon SD, Frye JG, et al. 2013. The intestinal fatty acid propionate inhibits Salmonella invasion through the post-translational control of HilD. Mol. Microbiol. 87: 1045-1060. 